\title{
2D Heterostructure for Enhanced Gas Barrier Performance via Synergetic Effect
}

\author{
Hayoung Ko ${ }^{\mathrm{a}, \mathrm{b}}$, Joo Song Lee ${ }^{\mathrm{a}, \mathrm{c}}$, and Soo Min Kim ${ }^{\mathrm{a}, *}$ \\ ${ }^{a}$ Institute of Advanced Composite Materials, Korea Institute of Science and Technology (KIST), Jeollabuk-do, 55324, Republic of Korea \\ ${ }^{b}$ Department of Energy Science, Sungkyunkwan University, Gyeonggi-do 16419, Republic of Korea \\ ${ }^{c}$ Applied Materials, Institute for BIN Convergence, Department of BIN Fusion Technology and Department of Polymer-Nano Science \\ and Technology, Chonbuk National University, Jeollabuk-do 54896, Republic of Korea
}

Received October 29, 2018; accepted November 8, 2018

\begin{abstract}
We report the dramatically enhanced gas barrier performance of polycrystalline monolayer graphene (PCG) film by the construction of 2D heterostructure with polycrystalline multilayered hexagonal boron nitride (PCMB) film, while maintaining high optical transmittance of $96.2 \%$ at $550 \mathrm{~nm}$. 2D heterostructure on flexible polyethylene terephthalate (PET) film is simply fabricated via a sequence transfer of PCMB and PCG grown by chemical vapor deposition (CVD). Water vapor transmission rate (WVTR) of individual PCG and PCMB films grown by chemical vapor deposition (CVD) exhibit 2.02 and $1.96 \mathrm{~g} / \mathrm{m}^{2} \cdot$ day, respectively, which is a slight improvement compared to that of bare PET substrate $\left(2.16 \mathrm{~g} / \mathrm{m}^{2} \cdot\right.$ day $)$ due to the imperfection of their crystal structure. In sharp contrast, 2D heterostructure consisted of PCG on PCMB shows almost 30 times lower WVTR value $\left(0.07 \mathrm{~g} / \mathrm{m}^{2} \cdot\right.$ day $)$ compared to that of PET substrate, which is the lowest reported value among CVD-grown 2D materials. The water vapor is impermeable in PCG stacked on thick and high-quality hBN grains, whereas water vapor only penetrates through structural defects such as grain boundaries or point defects in PCG on the grain boundaries of PCMB.
\end{abstract}

Keywords: Graphene, Hexagonal boron nitride, Chemical vapor deposition, Water vapor transmission rate, Water-barrier.

\section{Introduction}

Flexible and transparent electronics such as foldable and wearable display and skin-like thin flexible electronics have been developed for the next-generation devices [1]. To realize those unprecedented devices, organic materials as strong candidate have been developed due to their high quantum yield for light emission and high carrier mobility with lightweight, high transparency and flexibility [2]. However, organic materials are susceptible to the exposure of oxygen and water [3]. Specifically, water is much harmful to organic light-emitting diode (OLED) compared to oxygen [4]. Therefore, the encapsulation of these devices with impermeable gas barrier is highly required to ensure the permanence of the device.

Among several gas barrier materials $[5,6]$, hybrid organic and inorganic materials such as $\mathrm{Al}_{2} \mathrm{O}_{3} /$ parylene $\mathrm{C}$ have been suggested as flexible and transparent gas barriers and show superior water vapor barrier [7], but after several bending test, the performance of these gas barriers dramatically decreases due to the fracture of the inorganic materials [8].

*Corresponding author

E-mail: smkim@kist.re.kr
Hence, the development of alternative materials is timely important.

Recently, atomically thin two-dimensional (2D) layered materials have been proposed as an ideal flexible and transparent gas barrier due to their unique physical structure, high transparency and mechanical flexibility [9]. Among 2D materials, large-area monolayer graphene grown by chemical vapor deposition (CVD) has been widely investigated as a gas barrier [10,11]. But the gas barrier performance of graphene film is not as high as expected, owing to the presence of structural defects such as grain boundary and point defect in polycrystalline graphene (PCG) [12]. To improve the gas barrier performance, multistacked graphene film via a sequential transfer of PCG has been proposed [10]. While the gas permeability is reduced with increasing the number of graphene layers, the transparency of the gas barrier decreases, as an increment of the number of layers by a graphene's opacity of $2.3 \%$ [10]. Moreover, an improved crystallinity of graphene is obtained via a two-step graphene growth, resulted in the enhancement of gas barrier performance of monolayer graphene, but it is still not enough to meet technical requirements [13].

On the other hand, hexagonal boron nitride (hBN) 
consisted of alternating boron and nitrogen in hexagonal lattice is another 2D material which has an energy band gap of $\sim 6 \mathrm{eV}$. This ensures the optical absorbance in the visible light range is almost $0 \%$ regardless of its thickness. Recently, large-area and high-quality polycrystalline multilayered $\mathrm{hBN}$ (PCMB) film has been successfully synthesized on $\mathrm{Fe}$ foil [14]. While multilayer hBN grains exhibit similar crystallinity to single-crystal $\mathrm{hBN}$, grain boundaries exist between multilayer $\mathrm{hBN}$ grains. Thus far, the gas barrier performance of this PCMB film has not been investigated yet.

Here, we prove 2D heterostructure of PCG on PCMB film dramatically improves the gas barrier performance via the synergetic effect. 2D heterostructure is simply fabricated via a sequential transfer of monolayer PCG and PCBM onto polyethylene terephthalate (PET). While water vapor transmission rates (WVTR) of the individual PCG and PCMB films are slightly decreased compared to that of PET film, it is significantly decreased by $\sim 30$ times with the constructed 2D heterostructure. This might be attributed to significant reduction of the permeable region for water vapor transmission. Water vapor only penetrates through the structural defects in PCG on the grain boundaries of PCMB, whereas it is impermeable through thick and high-quality PCMB grains. Furthermore, a $96.2 \%$ transparency of the $2 \mathrm{D}$ heterostructure is attained at $550 \mathrm{~nm}$.

\section{Experimental}

\section{Growth of PCG and PCMB films}

PCG was grown on $\mathrm{Cu}$ foil (Alfa Aesar, $0.127 \mathrm{~mm}$, $99.9 \%$ ) by using low pressure CVD (LPCVD) [15]. To smoothen the $\mathrm{Cu}$ surface and remove native oxides, $\mathrm{Cu}$ foil was annealed at $1040{ }^{\circ} \mathrm{C}$ under $\mathrm{Ar}$ and $\mathrm{H}_{2}$ atmosphere at a flow rate of $100 \mathrm{sccm}$ for 1 hour and then PCG growth was conducted by introducing $\mathrm{CH}_{4}$ at a flow rate of $1.5 \mathrm{sccm}$ for $50 \mathrm{~min}$, while maintaining the initial flow rate of $\mathrm{H}_{2}$. After growth, the furnace was fast cooled to room temperature by opening the furnace under $\mathrm{H}_{2}$ atmosphere. PCMB film was grown on Fe foil (Alfa Aesar, $0.1 \mathrm{~mm}$, $99.99 \%$ ) by using LPCVD equipped with a bubbler system for supplying borazine precursor [14]. After annealing $\mathrm{Fe}$ foil under $\mathrm{H}_{2}$ atmosphere at a flow rate of 100 sccm at $1100{ }^{\circ} \mathrm{C}$ for 1 hour, PCMB was grown by introducing borazine at a flow rate of $0.8 \mathrm{sccm}$ for 1 hour while maintaining the initial flow rate of $\mathrm{H}_{2}$. After growth, the furnace was slowly cooled with a cooling rate of $5{ }^{\circ} \mathrm{C} /$ $\min$ to $700{ }^{\circ} \mathrm{C}$ under $\mathrm{H}_{2}$ atmosphere to induce the precipitation of multilayered $\mathrm{hBN}$ film [14].

\section{Transfer process}

Both PCG and PCMB films were transferred on PET film with supporting layer of polyethylene in cyclohexane (PMAX-6000, DongYang P-MAX Chemtech). First, polyethylene was spun onto the as-grown 2D materials on the metal substrates and then $\mathrm{Cu}$ and $\mathrm{Fe}$ foils were etched with $\mathrm{Cu}$ etchant (TYPE CE-100, TRANSENE) and Ni etchant (TFB, TRANENE), respectively. To remove any chemical residues, polyethylene/PCG (or PCMB) film was rinsed with fresh deionized water and transferred onto a PET film for WVTR measurement. Finally, polyethylene was removed in hot chlorobenzene at $180^{\circ} \mathrm{C}$ for $10 \mathrm{~min}$. To construct 2D heterostructure, polyethylene/PCG was transferred onto hBN/PET film and the polyethylene removed in hot chlorobenzene.

\section{Characterization}

The surface morphology and thickness of 2D film were characterized by optical microscopy (Eclipse LV150, Nikon) and scanning electron microscopy (SEM, Nova NanoSEM 450 , FEI). The quality of 2D film was characterized by Raman spectroscopy (In Via Raman spectroscopy, Renishaw, $514 \mathrm{~nm}$ LASER). Water vapor transmission was measured by WVTR measurement (Aquatran model2, MOCON).

\section{Results and discussion}

Figure 1 illustrates the fabrication process of WVTR sample and WVTR measurement. After transfer of 2D materials onto a PET film, 2D materials/PET was lapped with $\mathrm{Al}$ disk, which has a hole of $1 \mathrm{~cm}$ in diameter, for the penetration of water vapor, followed by the subsequent measurements of WVTR of the samples.

The quality of 2D materials was evaluated by SEM and Raman after the transfer of the samples on $\mathrm{SiO}_{2} / \mathrm{Si}$ substrate. SEM and optical images of PCG show highly uniform contrast over the whole region, indicating mostly monolayer graphene was grown [Figs. 2(a) and 2(b)]. On the other hand, the presence of multilayer $\mathrm{hBN}$ and its

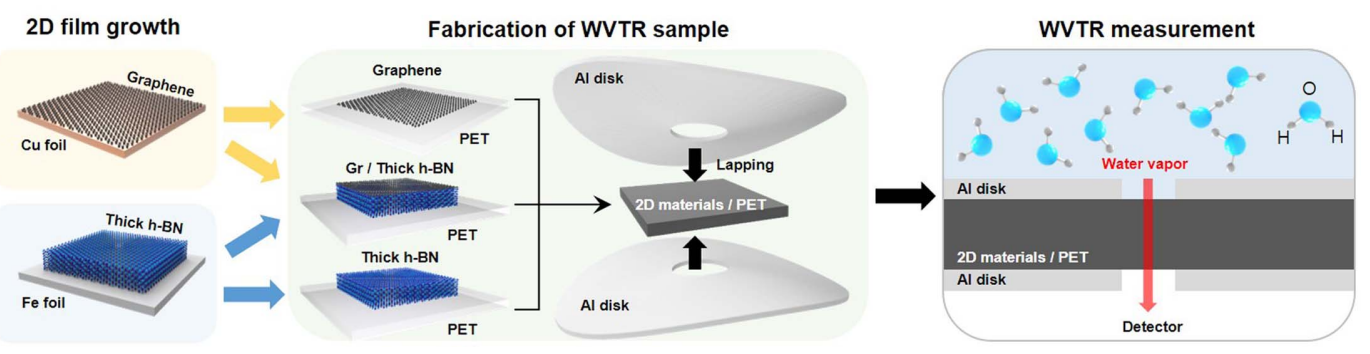

Figure 1. Schematic illustration of the fabrication procedure of 2D heterostructure gas barrier for WVTR measurement. 

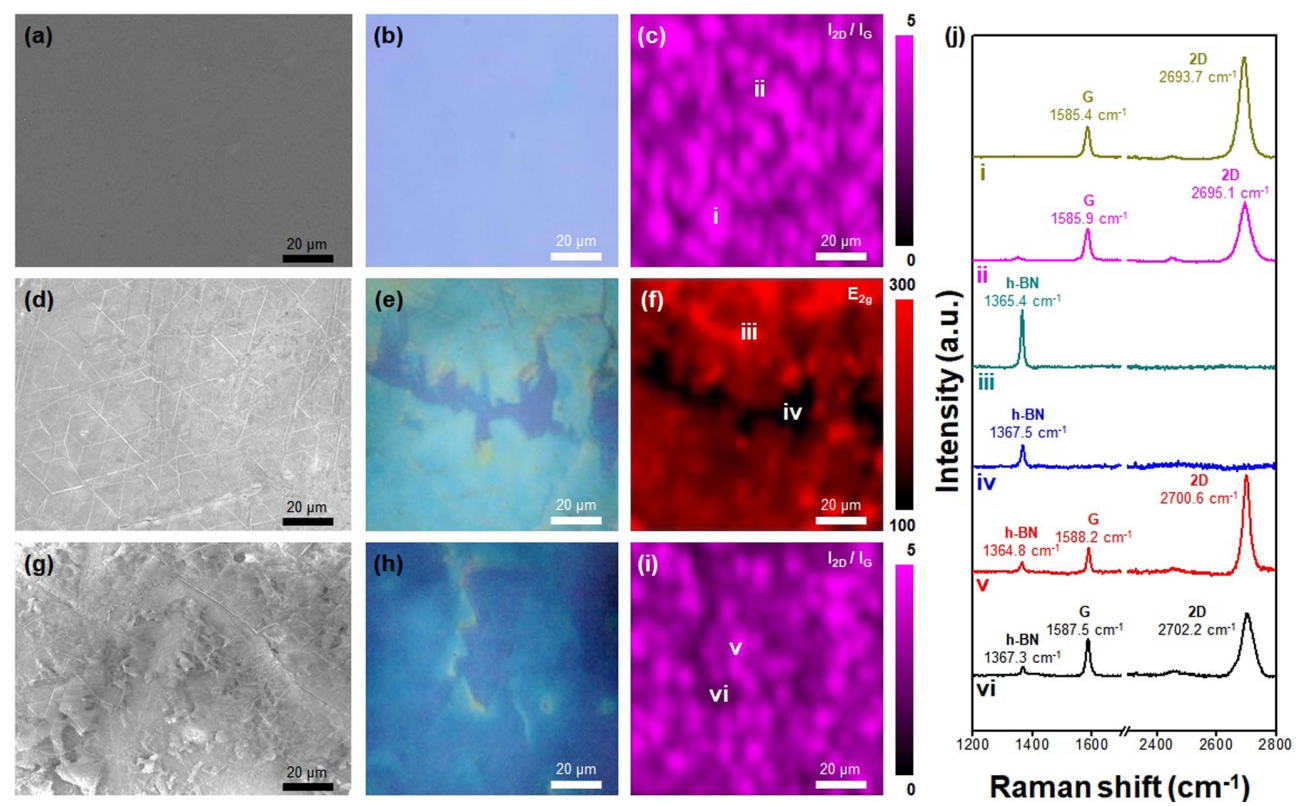

Raman shift $\left(\mathrm{cm}^{-1}\right)$

Figure 2. Characterization of 2D heterostructure. (a, d, g) SEM images of (a) PCG, (d) PCMB and (g) 2D heterostructure of PCG/PCMB on $\mathrm{SiO}_{2} / \mathrm{Si}$ substrate. (b, e, h) and (c, f, i) Optical and corresponding Raman mapping images of PCG, PCMB and 2D heterostructure, respectively. (j) Representative Raman spectrum of PCG, PCMB and PCG/PCMB from i, ii, iii, iv, v, vi spots in $\mathbf{c}, \mathbf{f}$ and $i$.

wrinkles are clearly observed in SEM and optical images [Figs. 2(d) and 2(e)] due to the precipitation of B and $\mathrm{N}$ out of Fe foil during cooling and thermal expansion coefficient difference between $\mathrm{hBN}$ and $\mathrm{Fe}$, respectively [14]. Furthermore, the thickness of $\mathrm{hBN}$ film is not uniform owing to different precipitation rate of $\mathrm{hBN}$ from the different $\mathrm{Fe}$ facets and grain boundaries of $\mathrm{Fe}$ foil [14]. The 2D heterostructure of PCG on PCMB is presented in the SEM and optical images, showing an overlap of the individual PCG and PCMB, implying that 2D heterostructure is well constructed via the sequential transfer [Figs. 2(g) and 2(h)]. To further analyze the presence of monolayer PCG and thickness uniformity of PCMB, Raman mapping was carried out. Raman spectra of graphene consisted of three representative phonon modes of D-band $\left(1350 \mathrm{~cm}^{-1}\right)$ related to defects, G-band $\left(1585 \mathrm{~cm}^{-1}\right)$ related to graphitic vibration and 2D-band $\left(2695 \mathrm{~cm}^{-1}\right)$ related to two-phonon inter-valley scattering at $\mathrm{K}$ point in Brillouin zone [16]. The intensity ratio of $2 \mathrm{D}$-band over G-band $\left(\mathrm{I}_{2 \mathrm{D}} / \mathrm{I}_{\mathrm{G}}\right)$ is typically attained to be more than 4 for monolayer graphene on $\mathrm{SiO}_{2} / \mathrm{Si}$ substrate [16]. The $\mathrm{I}_{2 \mathrm{D}} / \mathrm{I}_{\mathrm{G}}$ in Raman mapping image shows higher than 1 over the whole area [Fig. 2(c)], indicative of the presence of only monolayer graphene [17]. In addition, Raman intensity of $\mathrm{E}_{2 \mathrm{~g}}$ phonon near $1365 \mathrm{~cm}^{-1}$ for hBN increases as a function of number of layers [18]. The Raman mapping image for $\mathrm{E}_{2 \mathrm{~g}}$ phonon displays the variance of the intensity of $E_{2 g}$ peak depending on the position [Fig. 2(f)], implying that the thickness of $\mathrm{hBN}$ is randomly distributed at each position. For 2D heterostructure of PCG on PCMB, the Raman mapping image for $\mathrm{I}_{2 \mathrm{D}} / \mathrm{I}_{\mathrm{G}}$ shows uneven values due to the different thickness of hBN [Fig. 2(i)] [14]. Typically, graphene on thick $\mathrm{hBN}$ layer shows higher $\mathrm{I}_{2 \mathrm{D}} / \mathrm{I}_{\mathrm{G}}$ than that on $\mathrm{SiO}_{2} / \mathrm{Si}$ substrate, because thick hBN blocks the charge-transfer from $\mathrm{SiO}_{2} / \mathrm{Si}$ substrate [14]. But overall, $\mathrm{I}_{2 \mathrm{D}} / \mathrm{I}_{\mathrm{G}}$ is still higher than $\mathrm{PCG}$ on $\mathrm{SiO}_{2} / \mathrm{Si}$ substrate. Figure $2 \mathrm{~J}$ shows the representative Raman spectra from the region i, ii [PCG in Fig. 2(c)], iii, iv [PCMB in Fig. 2(f)], v and vi [2D heterostructure in Fig. 2(i)], respectively. The Raman spectrum of PCG shows high intensity of 2D-band with weak D-band intensity, indicative of the high-quality graphene [(i) in Fig. 2(j)]. But some regions show higher D-band than that of other region, leading to the lower 2Dband intensity [(ii) in Fig. 2(j)]. This might be attributed to the interruption of the tow-phonon inter-valley scattering, caused by the presence of the structural defects in PCG [16]. On the other hand, the full width at half maximum (FWHM) of $E_{2 \mathrm{~g}}$ peak at thick $\mathrm{hBN}$ is $\sim 11 \mathrm{~cm}^{-1}$, which is almost equivalent to that of single crystal hBN [(iii) in Fig. 2J(j)] [19]. This signifies extremely high crystallinity in this region. The typical grain size of $\mathrm{hBN}$ is few ten micrometers [Fig. 2(e)]. Furthermore, the FWHM of $E_{2 g}$ increases to $12 \mathrm{~cm}^{-1}$ at the regions of the grain boundaries or thin layer hBN [(iv) in Fig. 2(j)], implying that the crystallinity of $\mathrm{hBN}$ in this region is relatively poor. Furthermore, all the phonon modes of G-band, 2D-band and $\mathrm{E}_{2 \mathrm{~g}}$ from PCG and PCBM are clearly visible in the Raman spectra of 2D heterostructure [(v) and (vi) in Fig. 2(j)]. Interestingly, the $\mathrm{I}_{2 \mathrm{D}} / \mathrm{I}_{2 \mathrm{G}}$ is higher than that of PCG on thick hBN region [(v) in Fig. 2(j)], whereas it is weakened at PCG on thin layer or grain boundary of PCMB [(vi) in Fig. 2(j)]. This indicates that thick and high-quality hBN is more effective in blocking the charge transfer from $\mathrm{SiO}_{2} / \mathrm{Si}$ substrate compared to low quality $\mathrm{hBN}$. 

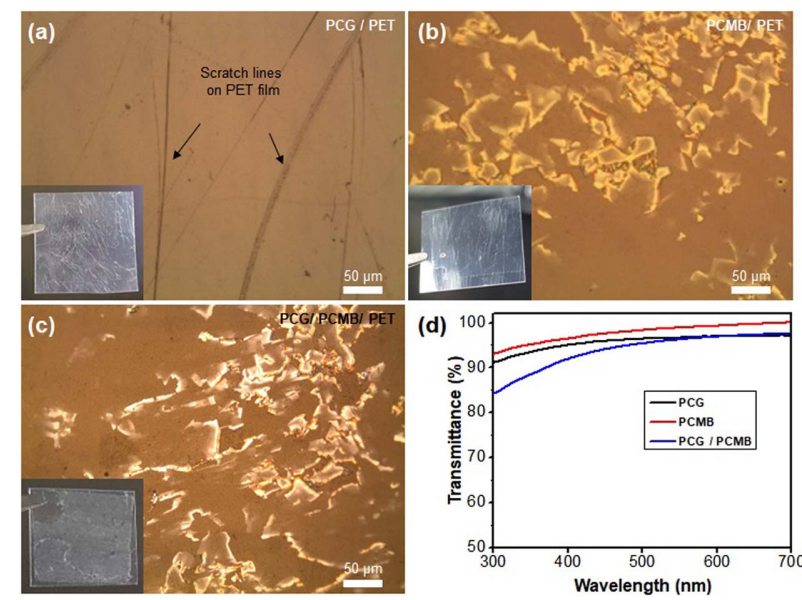

Figure 3. Transmittance of 2D gas barrier. (a-c) Optical image of (a) PCG (b) PCMB and (c) 2D heterostructure on PET substrate. Each inset shows the photograph of the sample. (d) Optical transmittance spectra of 2D gas barrier on glass substrate.

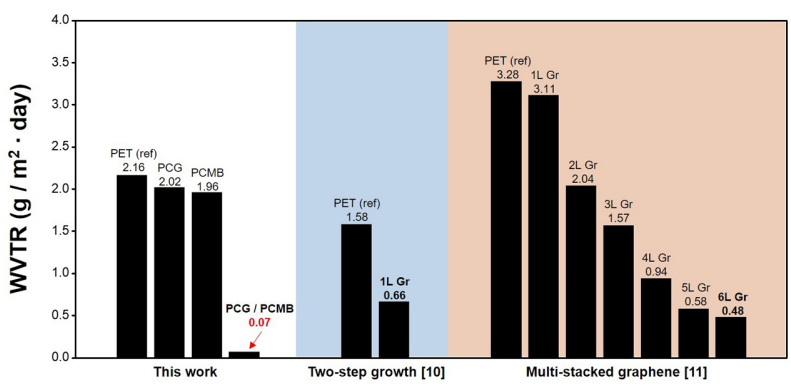

Figure 4. Comparison of WVTR with various 2D gas barriers.

After transfer of 2D materials on PET substrate for WVTR measurement, it is difficult to distinguish the presence of graphene [Fig. 3(a) and its inset]. Only the scratched regions are visible. On the other hand, thick hBN region in both PCMB and 2D heterostructure on PET can be observed in the optical images [Figs. 3(b) and 3(c)], whereas the transparency of both films looks comparable to that of bare PET film [Insets of Figs. 3(b) and 3(c)]. As mentioned above, a high transparent gas barrier is highly required for transparent electronic applications. Therefore, the transmittance of 2D materials after transfer onto a glass substrate was measured [Fig. 3(d)]. The transmittance of PCG at $550 \mathrm{~nm}$ is only $96.6 \%$, whereas it is $98.8 \%$ for PCMB. Furthermore, a transmittance of $96.2 \%$ is obtained for the $2 \mathrm{D}$ heterostructure. The transmittance of ideal monolayer and bilayer graphene is $97.7 \%$ and $95.4 \%$ with the monolayer graphene's opacity of $2.3 \%$ [20]. The obtained values are ranged between monolayer and bilayer. The slight reduction of transmittance might be attributed to polyethylene residues, used for the transfer process. These results prove that 2D heterostructure gas barrier exhibit high transmittance.

The WVTRs of bare PET film, PCG, PCMB and 2D heterostructure on PET film were obtained to be 2.16, 2.02, 1.96 and $0.07 \mathrm{~g} / \mathrm{m} \cdot$ day, respectively (Fig. 4). The WVTR was not much decreased by PCG and PCMB compared to that of PET. But it is significantly decreased by $\sim 30$ times for the 2D heterostructure on PET film, which is the highest reported value among CVD-grown 2D materials. The WVTR of 2D heterostructure obtained, is one order of magnitude lower than the values from the two-step graphene growth and multi-stacked graphene via a sequential transfer approach $[10,11]$.

To understand the effect of 2D heterostructure, the analysis of physical structure of 2D materials is of importance. Water vapor can penetrate through structure defects such as grain boundary or point defects, whereas it is impermeable to a perfect $2 \mathrm{D}$ crystal. Therefore, the grain size of graphene $\left(\mathrm{L}_{\mathrm{a}}\right)$ was extracted as $\mathrm{L}_{\mathrm{a}}(\mathrm{nm})=2.4 \times 10^{-10} \lambda_{\text {laser }}{ }^{4}$ $\left(\mathrm{I}_{\mathrm{D}} / \mathrm{I}_{\mathrm{G}}\right)^{-1}$, where $\lambda_{\text {laser }}$ and $\mathrm{I}_{\mathrm{D}} / \mathrm{I}_{\mathrm{G}}$ are the excitation energy of laser in Raman measurement and the intensity ratio of $\mathrm{D}$ band over G-band respectively [21]. The average of $L_{a}$ in PCG is obtained to be $200 \pm 50 \mathrm{~nm}$, indicating the presence of lots of grain boundaries in PCG film Thus, water vapor is easily permeable through those structural defects, resulting in the small reduction of WVTR value (Fig. 5). In addition, PCBM film which consists of well-crystallized thick grains with the size of few ten micrometers and lowquality few layers were combined at the grain boundaries. Even though water is impermeable through thick hBN grain, it can penetrate through grain boundaries of hBN like PCG. Hence, the WVTR value of PCMB is slightly lower than that of PCG. In contrast to the individual polycrystalline 2D materials, water vapor transmission can be greatly minimized by forming $2 \mathrm{D}$ heterostructure. Water vapor only penetrates through structure defects in PCG on grain boundaries of $\mathrm{hBN}$, whereas it is completely blocked at other regions by the presence of thick $\mathrm{hBN}$ grains.

\section{Conclusions}

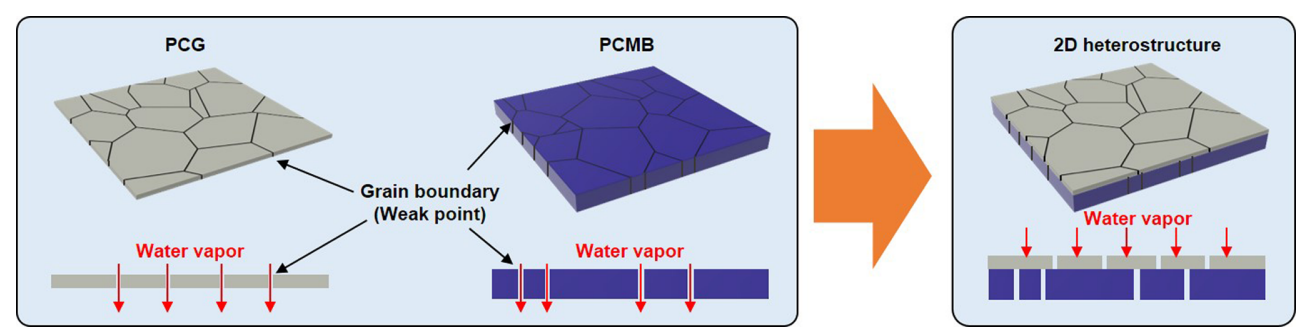

Figure 5. Illustration of the mechanism for enhanced gas barrier performance by the construction of 2D heterostructure. 
We have successfully developed a remarkable gas barrier by construction of $2 \mathrm{D}$ heterostructure with polycrystalline graphene and $\mathrm{hBN}$. 2D heterostructure was fabricated with a sequential transfer of PCG onto PCMB/PET substrate. Water vapor can only penetrate through structural defects such as grain boundaries or point defects, whereas it is completely blocked by the thick and high-quality $\mathrm{hBN}$ grains. By this synergetic effect, WVTR of $0.07 \mathrm{~g} / \mathrm{m}^{2}$-day is attained with a transmittance of $96.2 \%$ at $550 \mathrm{~nm}$, which is the highest reported value among CVD-grown $2 \mathrm{D}$ materials. This vertical 2D heterostructure gas barrier will not only advance flexible and transparent gas barrier, but also promote other 2D heterostructures for gas barrier applications.

\section{Acknowledgments}

This work is supported by the Basic Science Research Program through the National Research Foundation of Korea (NRF) funded by the Ministry of Science, ICT \& Future Planning (2018R1A2B2002859) and the Korea Institute of Science and Technology (KIST) Institutional Program.

\section{Reference}

[1] D. Akinwande, N. Petrone, and J. Hone, Nat. Commun. 5, 5678 (2014).

[2] A. Sugimoto, H. Ochi, S. Fujimura, A. Yoshida, T. Miyadera, and M. Tsuchida, IEEE. J. Sel. Top. Quant. 10, 107 (2004).

[3] M. Schaer, F. Nu esch, D. Berner, W. Leo, and L. Zuppiroli, Adv. Funct. Mater. 11, 116 (2001).
[4] D. Yu, Y. Q. Yang, Z. Chen, Y. Tao, and Y. F. Liu, Opt. Commun. 362, 43 (2016).

[5] R. K. Bharadwaj, Macromolecules. 34, 9189 (2001).

[6] C. Shanchez, B. Julián, P. Belleville, and M. Popall, J. Mater. Chem. 15, 3559 (2005).

[7] X. Xie, L. Rieth, L. Williams, S. Negi, R. Bhandari, R. Caldwell, R. Sharma, P. Tathireddy, F. Solzbacher, and J. Neural Eng. 11, 026016 (2014).

[8] W. Xiao, D. Y. Hui, C. Zheng, D. Yu, Y. Y. Qiang, C. Ping, C. L, Xiang, and Z. Yi, Nanoscale. Res. Lett. 10, 130 (2015).

[9] K. S. Novoselov, V. I. Fal'ko, L. Colombo, P. R. Gellert, M. G. Schwab, and K. Kim, Nature 490, 190 (2012).

[10] K. Choi, S. Nam, Y. Lee, M. Lee, J. Jang, S. J. Kim, Y. J. Jeong, H. Kim, S. Bae, J. B. Yoo, S. M. Cho, J. B. Choi, H. K. Chung, J. H. Ahn, C. E. Park, and B. H. Hong, ACS Nano. 9, 5818 (2015).

[11] T. H. Seo, S. Lee, H. Cho, S. Chandramohan, E. K. Suh, H. S. Lee, S. K. Bae, S. M. Kim, M. Park, J. K. Lee, and M. J. Kim, Sci. Rep. 6, 24132 (2016).

[12] D. Ding, H. Hibino, H. Ago, J. Phys. Chem. C. 122, 902 (2018).

[13] T. V. Duncan, J. Colloid Interf. Sci. 363, 1 (2011).

[14] S. M. Kim, A. Hsu, M. H. Park, S. H. Chae, S. J. Yun, J. S. Lee, D. H. Cho, W. Fang, C. Lee, T. Palacios, M. Dresselhaus, K. K. Kim, and Y. H. Lee, Nat. Commun. 6, 8662 (2015).

[15] X. Li, W. Cai, J. An, S. Kim, J. Nah, D. Yang, R. Piner, A. Velamakanni, I. Jung, E. Tutuc, S. K. Banerjee, L. Colombo, and R. S. Ruoff, Science 324, 1312 (2009).

[16] A. C. Ferrari, M. V. Scardaci, C. Casiraghi, M. Lazzeri, F. Mauri, S. Psicanec, D. Jiang, K. S. Novoselov, S. Roth, and A. K. Geim, Phys. Rev. Lett. 97, 187401 (2006).

[17] Y. Y. Wang, Z. H. Ni, T. Yu, Z. X. Shen, H. M. Wang, Y. H. Wu, W. Chen, and A. T. S. Wee, J. Phys. Chem. 112, 10637 (2008).

[18] R. Geick, C. H. Perry, and G. Rupprecht, Phys. Rev. 146, 543 (1966).

[19] Y. Kubota, K. Watanabe, O. Tsuda, and T. Taniguchi, Science 317, 932 (2007).

[20] W. B. Cho, J. W. Kim, H. W. Lee, S. Bae, B. H. Hong, S. Y. Choi, I. H. Baek, K. Kim, D. I. Yeom, and F. Rotermund, Opt. Lett. 36, 4089 (2011).

[21] M. A. Pimenta, G. Dresselhaus, M. S. Dresselhaus, L. G. Cancado, A. Jorio, and R. Saito, Phys. Chem. Chem. Phys. 9, 1276 (2007). 